\title{
PHASE BEHAVIOR OF LIGHT GAS MIXTURES \\ AT HIGH PRESSURES
}

\author{
WILLIAM B. STREETT
}

Science Research Laboratory and Department of Chemistry, U.S. Military Academy, West Point, N.Y., U.S.A.

\begin{abstract}
If solid surfaces exist beneath the visible clouds of the major planets, they may be expected to exist at depths and pressures at which the component gas mixtures solidify under their own weight. The elucidation of phase behavior in mixtures of light gases at very high pressures is therefore essential to the solution of the problem of deep atmosphere structures in these planets. Available experimental evidence suggests several possible extrapolations of the $\mathrm{H}_{2}$ - $\mathrm{He}$ phase diagram to high pressures. These have been used to develop a structural model for a $\mathrm{H}_{2}-\mathrm{He}$ atmosphere. In this model, gravitational separation of coexisting phases results in a layered structure, and it is shown that masses of $\mathrm{H}_{2}$-rich solid can exist in dynamic and thermodynamic equilibrium with a fluid layer of equal density but higher He content. This model forms the basis of a new hypothesis for Jupiter's Red Spot.
\end{abstract}

\section{Introduction}

It is generally accepted (DeMarcus, 1958; Öpik, 1962; Peebles, 1964) that Jupiter and Saturn are composed almost entirely of $\mathrm{H}_{2}$ and $\mathrm{He}$. If solid surfaces exist beneath the visible clouds of these planets, they may be expected to exist at depths and pressures at which the $\mathrm{H}_{2}$ - $\mathrm{He}$ mixture solidifies under its own weight. Whether solidification occurs depends on the equation of path of the atmosphere and the melting behavior of the mixture at high pressures and temperatures. Neither is known with any certainty, so the question of whether a solid surface exists remains open.

\section{Solidification of Gas Mixtures}

Existing models for the interiors of Jupiter and Saturn (DeMarcus, 1958; Opik, 1962) have been developed on the assumption that the presence of $\mathrm{He}$ has little or no effect on fluid-solid phase transitions in the molecular phase of $\mathrm{H}_{2}$. In this case, solidification occurs abruptly at the point at which the equation of path of the atmosphere crosses the melting curve of hydrogen (Point (a) in Figure 1). Solid and fluid phases coexist only at this point, which marks the level of the planet's solid surface. The purpose of this paper is to point out that the presence of He adds a second degree of freedom (in the context of the phase rule) to the conditions of equilibrium between two phases in a $\mathrm{H}_{2}-\mathrm{He}$ mixture, and that this may produce a more complicated structure in the region of transition from fluid to solid in a $\mathrm{H}_{2}-\mathrm{He}$ body.

Thermodynamic equilibrium between solid and fluid phases in a $\mathrm{H}_{2}-\mathrm{He}$ mixture is likely to exist over a finite distance along the equation of path (effectively over a range of depths in the atmosphere) with the compositions of the coexisting phases varying continuously with pressure and temperature in this region. This behavior is illustrated in the $P-T$ diagram of Figure 2, which shows (schematically) the melting

Sagan et al. (eds.), Plane'tary Amospheres, $363-370$.

All Rights Reserved. (opyright i 1971 by the l.A.l. 


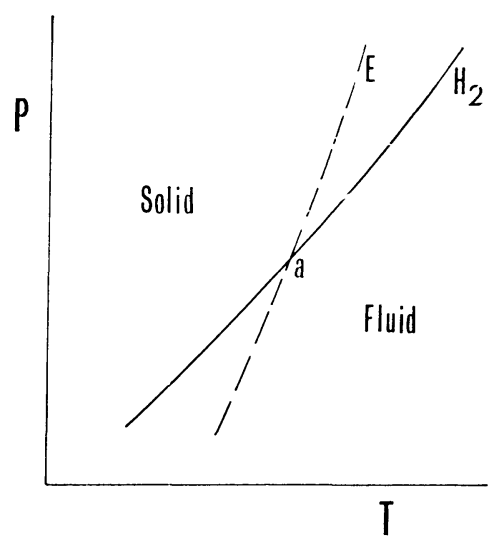

Fig. 1. Pressure-temperature diagram showing the melting curve of hydrogen $\left(\mathrm{H}_{2}\right)$ and the equation of path $(E)$ for a planetary atmosphere.

curves of $\mathrm{H}_{2}$ and $\mathrm{He}$, along with several hypothetical equations of path for the planetary atmosphere. The coexistence region for solid and fluid phases, in a $\mathrm{H}_{2}-\mathrm{He}$ mixture, lies approximately between the melting curves, so that for an equation of path $E_{1}$ (the isothermal case) these phases coexist approximately between points a and $\mathrm{b}$.*

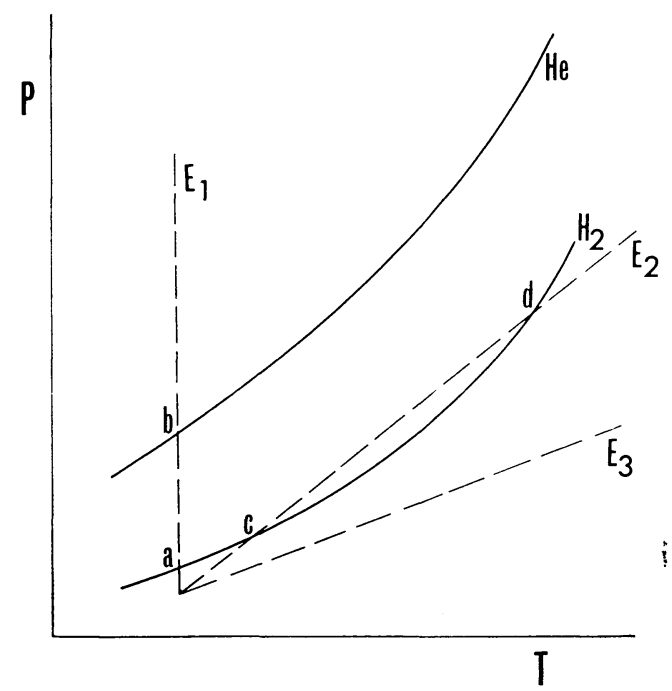

Fig. 2. Pressure-temperature diagram showing the melting curves of hydrogen and helium $\left(\mathrm{H}_{2}\right.$ and $\mathrm{He})$, and several equations of path $\left(E_{1}, E_{2}, E_{3}\right)$ representing different temperature gradients in a planetary atmosphere.

* The limiting boundaries, in $P-T$ space, for two-phase regions in a two-component system are defined by lines representing conditions of a single degree of freedom. These include three phase lines and critical lines, in addition to the pure component phase boundaries (melting curves, etc.) shown in Figure 1. A more complete discussion of phase diagrams in this context has been presented elsewhere (Streett, 1969; Streett and Hill, 1970). 
The planet would be entirely solid at pressures above $\mathrm{b}$ and fluid at pressures below $\mathrm{a}$. Lines $E_{2}$ and $E_{3}$ represent equations of path for which there are temperature gradients. If the gradient is large, as in $E_{3}$, solidification does not occur, and the planet remains fluid throughout. For the intermediate case, $E_{2}$, a region c-d exists in which solid and fluid phases may coexist (in thermodynamic equilibrium), while the planet is entirely fluid above and below.

\section{Gravitational Separation of Solid Fluid Phases}

Within the region of phase separation, coexisting phases would tend to separate in the gravitational field, producing a layered structure. At first glance, it seems natural to assume that the solid phase, being more dense than the fluid, would sink to the solid surface. A closer examination, however, suggests that this is not necessarily the case for a mixture of $\mathrm{H}_{2}$ and $\mathrm{He}$. In the case of a pure substance, such as $\mathrm{H}_{2}$, solidification is accompanied by a slight increase in density as a result of the more efficient packing of the molecules in an ordered lattice structure. Although a similar density increase results from solidification in a mixture, the relative densities of the two phases will be determined largely by their compositions, due to the large difference in the densities of the pure components. The $\mathrm{H}_{2}-\mathrm{He}$ system belongs to an unusual class of binary mixtures, in which the more volatile component $(\mathrm{He})$ has the higher molecular weight, and is therefore more dense, at high pressures, than the second component, even though the former may be a gas and the latter a solid or liquid in the pure state. (The estimated density of $\mathrm{He}$ at high pressures exceeds that of $\mathrm{H}_{2}$ by a factor of 3 or more (DeMarcus, 1958).) The result is that a reversal in the sign of the density difference between two coexisting phases occurs with increasing pressure at a fixed temperature. This behavior - known as the barotropic phenomenon - was first observed for gasliquid mixtures of $\mathrm{H}_{2}-\mathrm{He}$ by Kamerlingh-Onnes (1906). He observed that if a gas-liquid mixture of $\mathrm{H}_{2}-\mathrm{He}$ is compressed at a temperature of about $20 \mathrm{~K}$, the density of the gas phase (which is mostly $\mathrm{He}$ ) exceeds that of the liquid phase (which is mostly $\mathrm{H}_{2}$ ) at pressures above about $30 \mathrm{~atm}$. As the pressure passes through this value, the liquid phase rises up and floats on top of the gas phase. Similar behavior has been observed in other binary mixtures in which the more volatile component has the higher molecular weight, such as $\mathrm{N}_{2}-\mathrm{NH}_{3}$ (Krichevskii, 1940) and $\mathrm{CO}_{2}-\mathrm{H}_{2} \mathrm{O}$ (Takenouchi and Kennedy, 1964).

\section{Phase Behavior of $\mathrm{H}_{2}$-He Mixtures at High Pressures}

Although the behavior described above has not been observed experimentally in gas-solid mixtures of $\mathrm{H}_{2}-\mathrm{He}$, it almost certainly does occur. The equality of density between two phases in a two-component system reduces the number of degrees of freedom to one, and the locus of equal density points appears as a line on the $P-T$ diagram of the system. The probable location of this line can be found if the shape of the phase boundary curves in pressure-composition space are known. Although experimental data on the phase behavior of light gas mixtures at high pressures are meager, 
the available evidence (Tsiklis, 1946; Streett and Hill, 1970) suggests that the isothermal pressure-composition $(P-X)$ diagram for a mixture of $\mathrm{H}_{2}-\mathrm{He}$ will have the form shown in Figure 3.* Area $F$ is a region in which a single homogeneous fluid phase exists, while $S_{1}$ and $S_{2}$ are regions in which a single solid phase exists. $S_{1}$ is a $\mathrm{H}_{2}$-rich solid and $S_{2}$ a He-rich solid. The remaining areas $\left(S_{1}+F, S_{2}+F\right.$, and $\left.S_{1}+S_{2}\right)$ are regions in which two phases coexist in equilibrium. The behavior illustrated in this diagram is that of a binary mixture in which the components are completely miscible in the fluid phase $(F)$, but solidify to form partially miscible solid phases $\left(S_{1}\right.$ and $\left.S_{2}\right)$.

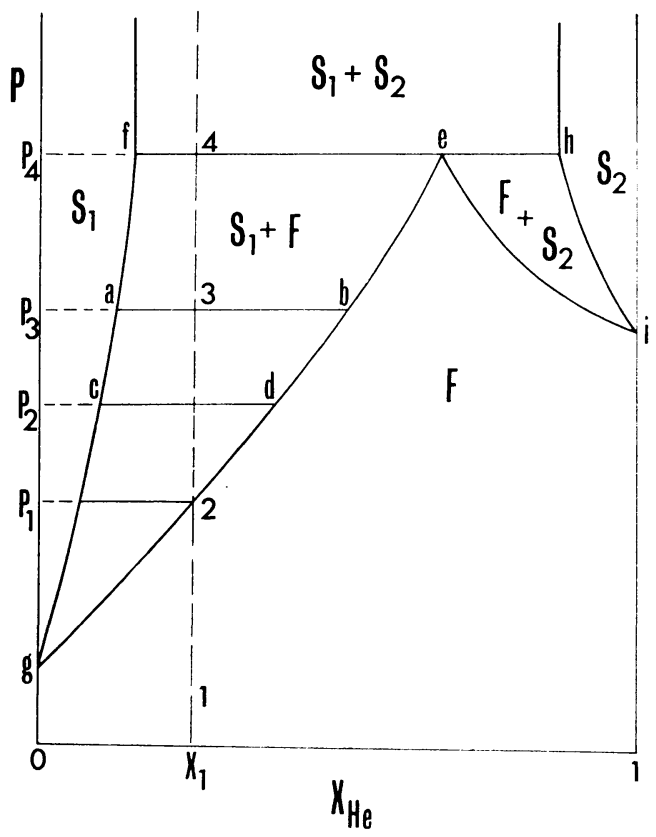

Fig. 3. Suggested pressure-composition diagram for $\mathrm{H}_{2}-\mathrm{He}$ mixtures at constant temperature.

\section{Proposed Structure for $\mathrm{H}_{2}-\mathrm{He}$ Atmosphere}

The sequence of thermodynamic states encountered in descending into the atmosphere of a body of overall composition $X_{1}$ would lie along an ascending vertical line 1-2-3-4 in Figure 3. At pressures below $P_{1}$ (point 2) a homogeneous fluid phase exists, while at pressures above $P_{4}$ (point 4) a mixture of two solid phases exists. $P_{4}$ therefore corresponds to the approximate depth of the solid surface. At any pressure between $P_{1}$ and $P_{4}$ the mixture separates into solid and fluid phases whose compositions are found at points where a horizontal line, at that pressure, intersects the phase boundary lines g-c-a-f and g-d-b-e. At pressure $P_{3}$, for example, the mixture of overall composition $X_{1}$ separates into a solid phase a and fluid phase b. It is of interest to consider

* There is some evidence (Sneed et al., 1968; Streett and Hill, 1970) that $\mathrm{H}_{2}-\mathrm{He}$ mixtures may separate into two distinct fluid phases at high pressures. This would result in a more complicated phase diagram and would lead to a more complex atmospheric structure (Streett, 1969). 
the relative densities of the coexisting phases defined by the lines g-c-a-f and g-d-b-e. At pressures just above $\mathrm{g}$ (the melting pressure of pure $\mathrm{H}_{2}$ ) the fluid phase is only slightly richer in $\mathrm{He}$ than the solid phase, and the latter is likely to be more dense by virtue of the volume decrease accompanying solidification. At higher pressures, however, the fluid phase is likely to be more dense by virtue of its higher $\mathrm{He}$ content. An example will illustrate this point. At a pressure of 10 kilobars DeMarcus (1958) estimates the molar volumes of cold $\mathrm{H}_{2}$ and $\mathrm{He}$ to be 7.3 and $4.3 \mathrm{~cm}^{3} /$ mole respectively. If these values are taken for the component molar volumes in the solid phase, and values, say, $5 \%$ greater for the fluid phase, it can be shown that the fluid will be more dense than the solid if its He content is greater by about fifteen mole percent. Then there must exist a pressure $P_{2}$ - the barotropic pressure - at which the coexisting phases $\mathrm{c}$ and $\mathrm{d}$ have equal densities. At pressures below $P_{2}$ the solid is more dense, and above $P_{2}$ the fluid is more dense. In this situation the solid phases, which condense

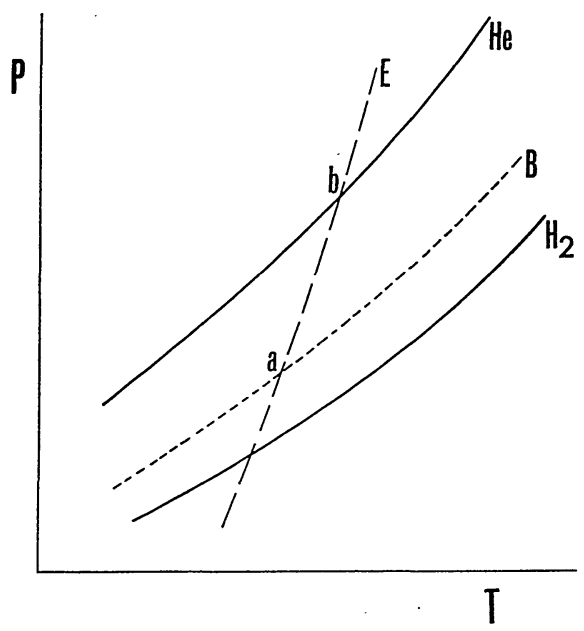

Fig. 4. Pressure-temperature diagram showing the melting curves of hydrogen and helium $\left(\mathrm{H}_{2}\right.$ and $\mathrm{He}$ ), the barotropic line $(B)$ at which the solid and fluid phases have equal density, and the equation of path $(E)$ of a planetary atmosphere.

between $P_{1}$ and $P_{4}$, will gravitate toward the level corresponding to $P_{2}$, both from above and below. Through slow diffusion within these solids, their compositions would eventually reach the value $c$ and they would remain suspended in dynamic and thermodynamic equilibrium with the surrounding fluid of composition $d$. In other words, the solid phase would behave as a thermodynamic Cartesian diver.

Consideration of the relative densities and compositions of the phases, and the effect of gravity, leads to an equilibrium structure with the following characteristics:

(1) the atmosphere above the level of $P_{2}$ has a uniform composition d;

(2) masses of $\mathrm{H}_{2}$-rich solid, of composition c, float at the level corresponding to $P_{2}$, in equilibrium with the fluid $\mathrm{d}$;

(3) at low levels, the fluid composition varies with depth and pressure along the lines d-b-e; and

25-P.A. 
(4) at the level corresponding to $P_{4}$ a solid surface exists, consisting of a mixture of two solid phases $f$ and $h$, whose average density exceeds that of the fluid $e$ in contact with it.

This structure has been derived for the special case of an isothermal atmosphere; however, because the phase diagram is likely to have the same form at higher temperatures, the same structure might exist in the presence of a temperature gradient. The locus of points at which the solid and fluid phases have equal densities - the barotropic curve - would appear as a line in the $P-T$ diagram, approximately parallel to, and lying above, the melting curve of $\mathrm{H}_{2}$, as shown in Figure 4. For an equation of path such as $E$, floating masses of $\mathrm{H}_{2}$-rich solid exist at the level of point a, and the solid surface lies in the vicinity of $b$. Extrapolations of the melting curves of $\mathrm{H}_{2}$ and $\mathrm{He}$ to high pressures suggest that, for temperatures of the order of a few hundred degrees, point a in Figure 4 would lie at a depth of about $800 \mathrm{~km}$ and point $\mathrm{b}$ at a depth of

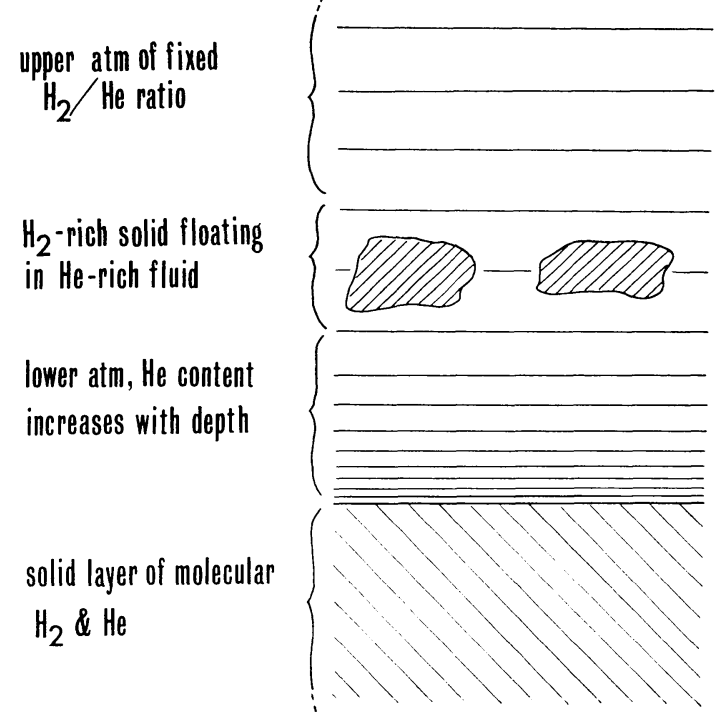

Fig. 5. Proposed structure for $\mathrm{H}_{2}-\mathrm{He}$ atmosphere, based on the phase diagram of Figure 3 . about $1600 \mathrm{~km}$, below the visible surface of Jupiter. A graphic picture of the structure suggested here is shown in Figure 5. The relevance of this picture to the floating raft concept of the Red Spot is immediately obvious.

\section{A New Hypothesis for Jupiter's Red Spot}

The structure suggested by Figure 5 forms the basis of a new hypothesis - called the Cartesian diver hypothesis - which seeks to explain the physical nature and observed variations in longitude, size, and intensity of the Red Spot. The following is a brief summary of this hypothesis; full details will be published elsewhere (Streett et al., 1971). 
According to the Cartesian diver hypothesis, the Red Spot is a region of contrast in the cloud structure of Jupiter's outer layers, caused by the presence of a mass of $\mathrm{H}_{2}$-rich solid (the diver) floating within a fluid layer of $\mathrm{H}_{2}$ and $\mathrm{He}$ at some depth below the visible surface. The resulting physical model thus includes some of the qualitative features of both a floating raft and a Taylor column. It can be shown that the floating solid behaves as a thermodynamically stabilized Cartesian diver: that is, as a floating object which seeks an equilibrium level within a fluid layer. In the case of the Red Spot the floating solid is not necessarily continuous, and, indeed, it is unlikely to be do.

Equations of motion of a stable Cartesian diver in a rotating system suggest that the longitudinal motion of the Red Spot consists of several periodic components of different amplitudes and frequencies. These predictions are in agreement with the conclusions of Solberg (1968) based on recent precise measurements of Red Spot longitude. When the effects of rapid rotation of the planet upon the dynamic behavior of the fluid in the vicinity of the Cartesian diver are included, the resulting physical model provides a qualitative explanation not only for the observed variations in size and intensity of the Red Spot, but also for the manner in which these variations correlate with changes in longitude.

\section{Conclusions}

Only a few of the more obvious consequences of phase separations in $\mathrm{H}_{2}$ - $\mathrm{He}$ mixtures at high pressures have been considered here. From a chemical standpoint, the most pressing need is for experimental data on the phase behavior and equation of state of $\mathrm{H}_{2}$-He mixtures at high pressures. The available data for this system (Sneed et al., 1969) are insufficient even to suggest a choice between several possible extrapolations of the phase diagram to high pressures. It is clear that knowledge of the phase behavior of $\mathrm{H}_{2}$-He mixtures at high pressures will be valuable, not only for understanding the deep atmosphere structures, but also for interpreting correctly some of the direct observational data for the upper atmosphere. We are preparing to carry out experimental studies of phase behavior in $\mathrm{H}_{2}-\mathrm{He}$ mixtures at pressures up to 10 kilobars. The results will be reported in due course.

\section{Acknowledgment}

The author is indebted to R. Wildt of Yale University for several useful discussions relating to this work.

\section{References}

DeMarcus, W. C.: 1958, Astron. J. 63, 2.

Kamerlingh-Onnes, H. and Keesom, W. H.: 1906, Comms. Phys. Lab. Univ. Leiden, No. 96a, 96b, 96c.

Krichevskii, I. R.: 1940, J. Phys. Chem. (U.S.S.R.) 12, 480.

Öpik, E. J.: 1962, Icarus 1, 200. 
Peebles, P. J. E.: 1964, Astrophys. J. 140, 328.

Peek, B. M.: 1958, in The Planet Jupiter, Faber and Faber, London, Chapter 19.

Reese, E. J. and Solberg, H. G.: 1969, Latitude and Longitude Measurements of Jovian Features in 1967-68, U.S. Government Research and Development Report N69-26410.

Sneed, C. M., Sonntag, R. E., and Van Wylen, G. J.: 1968, J. Chem. Phys. 49, 2410.

Solberg, H. G.: 1968, Icarus 8, 82.

Streett, W. B.: 1969, J. Atmospheric Sci. 26, 924.

Streett, W. B. and Hill, J. L. E.: 1970, in J. Chem. Phys. 52, 1402.

Streett, W. B., Ringermacher, H. I., and Veronis, G.: 1971, submitted to Icarus.

Takenouchi, S. and Kennedy, G. C.: 1964, Am. J. Sci. 262, 1055.

Tsiklis, D. S.: 1946, J. Phys. Chem. (U.S.S.R.) 20, 181.

\section{Question following Dr. Streett's paper}

Question: Can you state more accurately the pressures at which solids might be floating about in Jupiter's atmosphere?

Streett: No. In addition to our ignorance about the melting behavior of $\mathrm{H}_{2}$ and $\mathrm{He}$ at high pressures and temperatures, we have very little information about temperatures of the regions beneath the clouds which make up Jupiter's visible surface. For temperatures of the order of, say, $1000 \mathrm{~K}$, the estimated melting pressures of $\mathrm{H}_{2}$ and $\mathrm{He}$ are 460 kilobars and 800 kilobars, but these are based on extrapolations of experimental data which extend to temperatures of only about $100 \mathrm{~K}$ and pressures of about 20 kilobars. 